\title{
mTOR activation in medullary thyroid carcinoma with RAS mutation
}

\author{
Joana Lyra ${ }^{1,2}$, João Vinagre², Rui Batista ${ }^{2}$, Vasco Pinto ${ }^{1,2}$, Hugo Prazeres ${ }^{2,3}$, \\ Fernando Rodrigues ${ }^{4}$, Catarina Eloy ${ }^{2}$, Manuel Sobrinho-Simões ${ }^{1,2,5}$ and \\ Paula Soares ${ }^{1,2}$
}

${ }^{1}$ Medical Faculty, University of Porto, Al. Prof. Hernâni Monteiro, P-4200 Porto, Portugal, ${ }^{2}$ Institute of Molecular Pathology and Immunology of the University of Porto (Ipatimup) - Cancer Biology, RuaDr. Roberto Frias, s/n, 4200-465 Porto, Portugal, ${ }^{3}$ Molecular Pathology Service of the Portuguese Institute of Oncology of Coimbra FG, EPE, Av. Bissaya Barreto, 98, 3000-075 Coimbra, Portugal, ${ }^{4}$ Endocrinology Service of the Portuguese Institute of Oncology of Coimbra FG, EPE, Av. Bissaya Barreto, 98, 3000-075 Coimbra, Portugal, ${ }^{5}$ Department of Pathology, Hospital de S. João, Al. Prof. Hernâni Monteiro, P-4200 Porto, Portugal

Correspondence should be addressed to P Soares Email psoares@ipatimup.pt

\section{Abstract}

Objective: Rearranged during transfection (RET) mutations are well-known genetic events in sporadic and familial medullary thyroid carcinoma (FMTC). The presence of RAS mutations in sporadic cases, challenging the RET paradigm in these tumors, has been recently reported. We intend to evaluate mTOR pathway activation in RET- and RAS-mutated MTC. Materials and methods: In this study, we analysed the presence of RET, H-RAS, and K-RAS mutations in a series of 87 MTCs (82 apparently sporadic and five FMTCs; five apparently sporadic MTCs were eventually found to be familial). We also evaluated mTOR activation - using the expression of its downstream effector phospho-S6 ribosomal protein ( $p$ - 56 ) and the expression of the mTOR inhibitor, phosphatase and tensin homologue deleted on chromosome 10 (PTEN) - by immunohistochemistry.

Results: Our results revealed that RET mutations were present in $52.9 \%$ of the cases (46/87) and RAS mutations in $12.6 \%$ (11/87) of the whole series of MTCs and $14.3 \%$ of the 77 sporadic MTCs. The presence of RET and RAS mutations was mutually exclusive. RAS mutations were significantly associated with higher intensity of $\mathrm{p}$-S6 expression $(P=0.007)$, suggesting that the mTOR pathway is activated in such MTCs. We observed also an increased expression of p-S6 in invasive tumors $(P=0.042)$ and in MTCs with lymph node metastases $(P=0.046)$. Cytoplasmic PTEN expression was detected in $58.8 \%$ of the cases; cases WT for RAS showed a significantly lower expression of PTEN $(P=0.045)$.

Conclusions: We confirmed the presence of RAS mutation in $14.3 \%$ of sporadic MTCs and report, for the first time, an association between such mutations and the activation of the mTOR pathway. The evaluation of the mTOR activation by pS6 expression may serve as an indicator of invasive MTC.

\section{Introduction}

Medullary thyroid carcinoma (MTC) is a rare neuroendocrine tumor arising from the calcitonin-producing parafollicular $\mathrm{C}$ cells of the thyroid. It accounts for 5-8\% of all clinically evident thyroid tumors. MTCs can present as part of hereditary syndromes (20-25\%) (1) known as multiple endocrine neoplasia type 2 (MEN2): MEN2A, MEN2B, and familial MTC (FMTC). More frequently, MTCs present as sporadic cases (75-80\%). MTC is thought to be responsible for up to $14 \%$ of all thyroid cancerrelated deaths, distant metastases being the main cause in this setting (2). Treatment of patients with MTC is still difficult in many cases and alternative therapeutic options have been proposed $(2,3,4,5)$.

The most important genetic events contributing to MTC development are mutations in the proto-oncogene rearranged during transfection (RET). Germline RET
(C) 2014 European Society of Endocrinology Printed in Great Britain
Published by Bioscientifica Ltd. 
mutations are present in almost $100 \%$ of the hereditary cases, whereas $30-50 \%$ of sporadic MTC cases also display somatic RET mutations $(6,7)$. The most common mutations in the hereditary setting involve exon 10 (codons 609, 611, 618, and 620) or exon 11 (codon 634) in MEN2A; exon 16 (codon 918) - Met918Thr - in MEN2B; in FMTC families, mutations in exons $8,10,11,13,14$, and 15 partly overlap with the mutations detected in MEN2A $(4,8)$

According to Elisei et al. (9), p.Met918Thr somatic mutation was present in more than $50 \%$ of RET-mutated sporadic cases and it was associated with a worse prognosis (9). $40-60 \%$ of sporadic MTCs remained without a recognised genetic event $(10,11)$. Recently, Moura et al. (12) have reported the presence of RAS somatic mutations in $68 \%$ of $R E T$-negative sporadic MTCs (12). RAS mutations were only detected in $H-R A S$ and $K-R A S$ genes (none in $N$-RAS gene), apparently representing an alternative genetic event to RET mutations in sporadic MTC $(6,10$, $11,12,13,14)$. These results were reproduced by others $(10,11,13,14)$ with the percentage of $R A S$-mutated cases ranging from $0 \%$ (15) to $26.2 \%$ of MTCs (11). According to a meta-analysis by Ciampi et al. (11), the average prevalence of $R A S$ mutations in sporadic MTC is $8.8 \%$.

It has been demonstrated that the mTOR pathway is activated in sporadic and hereditary MTC $(13,15,16,17)$ and that RET mutations seem to play a role in the activation of such pathway in MTC $(4,6,15)$. It remains unknown whether RAS mutations can also lead to mTOR pathway activation in MTCs. It is also not known whether there are alterations of phospho-S6 ribosomal protein (p-S6) expression and other major components in this cascade, such as phosphatase and tensin homologue deleted on chromosome 10 (PTEN), in MTCs cases with RAS mutation (18).

In this study, we searched for mutations of RET, $H$-RAS, and $K-R A S$ in a series of 87 MTCs. We evaluated the mTOR activation in RET- and RAS-mutated MTCs using immunohistochemistry (IHC) for p-S6 (a downstream effector) and PTEN (a mTOR pathway inhibitor).

\section{Material and methods}

\section{Human cancer samples}

A total of 87 MTC samples from patients with apparently sporadic (AS-MTC, $n=82$ ) and familial $(n=5)$ MTCs were analysed by molecular and IHC methods. Formalinfixed, paraffin-embedded tissues were retrieved from the files of Centro Hospitalar S. João (HSJ)/Medical Faculty of Porto (FMUP)/Ipatimup (63 cases) and from the
Portuguese Institute of Oncology, Coimbra (IPO-C; 24 cases). The diagnosis of MTC was revised by M Sobrinho-Simões and C Eloy and confirmed by calcitonin staining. Clinicopathological and follow-up data were retrieved from the files of the Department of Pathology and Oncology of HSJ and from IPO databases (Table 1). Ipatimup cases included many consultation cases from which only limited demographic and clinical information was available. The study was approved by the Local Ethical Committee. National Ethical rules were followed in every procedure. In the cases suspected to be familial, peripheral blood samples were obtained with informed consent and genetic counselling was available for participants.

\section{DNA extraction, PCR, and Sanger sequencing}

DNA was obtained from formalin-fixed, paraffinembedded tissues (10-micron sections) after careful microdissection. DNA extraction was performed using the Ultrapep Tissue DNA Kit (AHN Biothecnologie, Nordhaussen, Germany) following the manufacturer's instructions.

Sanger's direct sequencing analysis of exons 10, 11, 13, 14,15 , and 16 of RET oncogene and exons 1 and 2 of both $H-R A S$ and $K-R A S$ was performed after specific amplification by PCR. Sequences of oligonucleotide primers to screen for RET hotspot mutations in the exons are mentioned above, and $H$-RAS and K-RAS hotspots can be found in Supplementary Table 1, see section on supplementary data given at the end of this article. Amplification

Table 1 Clinical and pathological data of MTC.

\begin{tabular}{|c|c|}
\hline & MTC \\
\hline \multicolumn{2}{|l|}{ Gender $(n=62)^{a}$} \\
\hline Female & $36(58.1 \%)$ \\
\hline Male & $26(41.9 \%)$ \\
\hline Age $(n=62 ; \text { mean } \pm \text { s.D. })^{a}$ & $53.2( \pm 14.3)$ \\
\hline Tumor size $(\mathrm{cm})(n=43 ; \text { mean } \pm \text { s.D. })^{a}$ & $2.61( \pm 1.74)$ \\
\hline \multicolumn{2}{|l|}{ Nodular metastasis $(n=31)^{\mathrm{a}}$} \\
\hline Absent & $10(32.3 \%)$ \\
\hline Present & $21(67.7 \%)$ \\
\hline \multicolumn{2}{|l|}{ Invasion $(n=30)^{\mathrm{a}}$} \\
\hline Absent & $5(16.7 \%)$ \\
\hline Present & $25(83.3 \%)$ \\
\hline \multicolumn{2}{|l|}{ Amyloid stroma $(n=46)^{a}$} \\
\hline Absent & $16(34.8 \%)$ \\
\hline Present & $30(65.2 \%)$ \\
\hline RET mutations $(n=87)$ & $46(52.9 \%)$ \\
\hline RAS mutations $(n=87)$ & $11(12.6 \%)$ \\
\hline RET and RAS WT $(n=87)$ & $30(34.5 \%)$ \\
\hline
\end{tabular}

${ }^{a}$ It was not possible to obtain complete clinicopathologic data from all the cases included in the series. 
of genomic DNA (25-100 ng) for RET was performed by PCR using the Qiagen Multiplex PCR Kit (Qiagen) according to the manufacturer's instructions. For $H$-RAS and $K-R A S$, we used PromegaGoTaq G2 Flexi DNA Polymerase (Promega). The sequencing reaction was performed with the ABI Prism BigDye Terminator Kit (Perkin-Elmer, Foster City, CA, USA), and the fragments were run in an ABI prism 3100 Genetic Analyser (PerkinElmer). The sequencing reactions were performed in a forward direction for all the exons, except for exon 2 of $H-R A S$, which was done in reverse direction. An independent PCR amplification/sequencing, in both directions, was performed in positive samples and in samples that were dubious. The genetic analysis was done first in tumoral tissue, and whenever a mutation was found, the germinative line was analysed in blood DNA or adjacent tissue DNA. We did not screen exon 16 for germline $R E T$ mutations because there was no clinical indication of MEN2B phenotype in the cases harbouring somatic mutation in this exon.

\section{Immunohistochemistry}

Immunohistochemistry for p-S6 and PTEN was performed in representative tumor tissue sections of 85 cases for PTEN and 84 cases for p-S6 (the remaining two and three cases, respectively, for PTEN and pS6, were excluded due to technical reasons). Briefly, deparaffinised and rehydrated sections were subjected to one of the antigen retrieval treatment methods, in a pressure cooker in $10 \mathrm{mM}$ sodium citrate buffer of $\mathrm{pH} 6.0$ (PTEN) or EDTA buffer of $\mathrm{pH} 9$ ( $\mathrm{p}$-S6) for antigen retrieval. The sections were incubated overnight at $4{ }^{\circ} \mathrm{C}$ in a humidified chamber with the primary antibody for PTEN (polyclonal, rabbit, 1:75) and p-S6 (polyclonal, mouse, 1:75), both from Cell Signaling Technology, Inc. (Danvers, MA, USA). The detection was obtained with a labelled streptavidinbiotin immunoperoxidase detection system (Thermo Scientific/Lab Vision, Fremont, CA, USA), and the immunohistochemical staining was developed with 3,3'-diaminobenzidine substrate. Omission of the primary antibody incubation was used as negative control. Previously tested samples of thyroid and pancreas were used as positive control. IHC evaluation was performed by two observers (C Eloy and J Lyra), independently. An IHC score was defined using the intensity score for expression (absent $=0$, faint $=1$, moderate $=2$, and strong $=3$ ) and the extension score of the expression $(0-5 \%=0, \quad 6-25 \%=1, \quad 26-50 \%=2, \quad 51-75 \%=3$, and $76-100 \%=4)$. We considered as 'low expression' when the p-S6 intensity score was 0 or 1, and as 'high expression' when p-S6 intensity score was 2 or 3. For PTEN it was also taken into account the cellular localisation of the staining (nuclear or cytoplasmic) and for IHC evaluation the same score as the one used for p-S6 was applied.

\section{Statistical analyses}

Statistical analysis was performed using 21.0 SPSS Statistical Package (SPSS, Inc., 2003). $\chi^{2}$, Fisher's exact test, and independent-samples $t$-test were performed in an attempt to correlate RAS and RET genotype results and clinicopathological data. The same statistical analysis was performed to evaluate immunohistochemical results (presented as intensity expression scores) and RET genotype, RAS genotype, and clinicopathological data. Values of $P \leq 0.05$ were considered statistically significant.

\section{Results}

\section{Genetic analysis}

In the 87 MTC cases screened for RET oncogene, $52.9 \%$ (46/87) were mutated: $9.2 \%(8 / 87)$ were mutated in exon $10,20.7 \%(18 / 87)$ in exon $11,1.1 \%(1 / 87)$ in exon 13 , $2.3 \%(2 / 87)$ in exon 15 , and 20.7\% (18/87) in exon 16 . We confirmed the germline nature of RET mutations in the five familial cases suspicious to be MEN2A and we detected five additional germline mutations in five apparently sporadic MTC. In the ten cases with germline mutations, there were seven $(70.0 \%)$ with mutations in exon 11, one in exon 10 (10.0\%), one in exon 13 (10.0\%), and one in exon 15 (10.0\%).

RAS mutations were detected in $12.6 \%(11 / 87)$ of the tumors: eight in exon 2 of HRAS - 9.2\% (8/87) - and three in exon 1 of $K R A S-3.4 \%$. A mutually exclusive pattern was observed between RET and RAS mutations; $R A S$ mutations were only present in RET-negative cases $(10,11,12)$. RAS mutations were found in $26.8 \%(11 / 41)$ of WT RET MTCs.

Patients with MTCs displaying germline RET mutations were younger than those with MTCs with a somatic RET mutation or no RET mutation at all (41.8 years vs 55.2 years, $P=0.008$ ). We did not find any other significant association between RET mutations and gender, tumor size, nodal metastasis, amyloid stroma, or invasive features. A similar analysis for RAS-mutated cases did not disclose any associations with any clinicopathological feature (Table 2). 
Table 2 Clinicopathologic data, $\mathrm{p}-\mathrm{S} 6$ expression, and RET and RAS mutational status.

\begin{tabular}{|c|c|c|c|c|c|c|c|c|c|}
\hline & \multicolumn{3}{|c|}{ RET } & \multicolumn{3}{|c|}{ RAS } & \multicolumn{3}{|c|}{ p-S6 expression ${ }^{a}$} \\
\hline & WT & Mutation $^{\mathrm{b}}$ & $P$ value & WT & Mutation & $P$ value & $\begin{array}{l}\text { Low expression, } \\
\qquad S \leq 1\end{array}$ & $\begin{array}{l}\text { High expression, } \\
\qquad S \geq 2\end{array}$ & $P$ value \\
\hline \multicolumn{10}{|l|}{ Gender } \\
\hline Female & $15(55.6 \%)$ & $21(60.0 \%)$ & 0.798 & $31(55.4 \%)$ & $5(83.3 \%)$ & 0.387 & $15(55.6 \%)$ & $19(59.4 \%)$ & 0.797 \\
\hline Male & $12(44.4 \%)$ & $14(40.0 \%)$ & & $25(44.6 \%)$ & $1(16.7 \%)$ & & $12(44.4 \%)$ & $13(40.6 \%)$ & \\
\hline Age & $57.1( \pm 13.9)$ & $50.2( \pm 13.9)$ & 0.059 & $52.7( \pm 13.3)$ & $58.5( \pm 22.4)$ & 0.345 & $55.8( \pm 13.4)$ & $51.8( \pm 15.1)$ & 0.286 \\
\hline Tumor size $(\mathrm{cm})$ & $2.3( \pm 1.7)$ & $2.8( \pm 1.8)$ & 0.358 & $2.6( \pm 1.8)$ & $3.3( \pm 1.1)$ & 0.603 & $2.6( \pm 1.6)$ & $2.9( \pm 1.8)$ & 0.561 \\
\hline \multicolumn{10}{|c|}{ Nodular metastasis } \\
\hline Absent & $3(30.0 \%)$ & $7(33.3 \%)$ & 1.000 & $10(33.3 \%)$ & $0(0 \%)$ & 1.000 & $9(50.0 \%)$ & $1(7.7 \%)$ & 0.046 \\
\hline Present & $7(70.0 \%)$ & $14(66.7 \%)$ & & $20(66.7 \%)$ & $1(100.0 \%)$ & & $9(50.0 \%)$ & $12(92.3 \%)$ & \\
\hline \multicolumn{10}{|l|}{ Invasion } \\
\hline Absent & $2(20.0 \%)$ & $3(15.0 \%)$ & 1.000 & $5(20.0 \%)$ & $0(0 \%)$ & - & $5(33.3 \%)$ & $0(0 \%)$ & 0.042 \\
\hline Present & $8(80.0 \%)$ & $17(85.0 \%)$ & & $25(80.0 \%)$ & $0(0 \%)$ & & $10(66.7 \%)$ & $15(100.0 \%)$ & \\
\hline \multicolumn{10}{|l|}{ Amyloid stroma } \\
\hline Absent & $6(35.3 \%)$ & $10(34.5 \%)$ & 1.000 & $15(34.9 \%)$ & $1(33.3 \%)$ & 1.000 & $11(44.0 \%)$ & $4(20.0 \%)$ & 0.118 \\
\hline Present & $11(64.7 \%)$ & $19(65.5 \%)$ & & $28(65.1 \%)$ & $2(66.7 \%)$ & & $14(56.0 \%)$ & $16(80.0 \%)$ & \\
\hline
\end{tabular}

$P$ values obtained from $\chi^{2}$ test realized for gender, nodular metastasis, invasion, and amyloid stroma and Independent $t$-test for age and tumor size. ${ }^{a}$ These tests were carried out by stratifying p-S6 intensity score in two groups - low expression (0 or 1$)$ and high expression (2 or 3 ).

bomatic or germline RET mutations.

\section{p-S6 expression in MTC}

Ribosomal protein S6 is part of the mTOR cascade and when phosphorylated it indicates upstream mTOR pathway activation. We assessed mTOR cascade activity by IHC using an antibody for p-S6. Out of the 84 cases evaluated for p-S6 expression, 53.6\% (45/84) had high intensity ( $S \geq 2)$ vs $46.4 \%$ (39/84) presenting low intensity $(S \leq 1)$ of p-S6 expression (Tables 2 and 3$)$.

We did not observe any significant difference in the intensity score of p-S6 expression between tumors with and without RET mutation (germinative or somatic).
Yet, a significant difference in the intensity score of p-S6 was observed in tumors harbouring RAS mutation in comparison with WT RAS cases $(P=0.009$; Table 3$)$. Furthermore, a Pearson's $\chi^{2}$ test comparing p-S6 expression groups was applied within the series of $R A S$-mutated cases and showed a statistically significant over-representation of p-S6 high expression cases $(90.9 \%$ of $R A S$-mutated cases vs $9.1 \%$ in WT RAS; $P=0.007)$.

The intensity of p-S6 expression was significantly associated with the presence of lymph node metastases $(P=0.046$; Table 2$)$. The intensity of $\mathrm{p}$-S6 expression was significantly higher in invasive than in non-invasive

Table 3 p-S6 and PTEN intensity expression according to the mutational status of MTC.

\begin{tabular}{|c|c|c|c|c|c|c|c|c|c|}
\hline & \multicolumn{3}{|c|}{ p-S6 expression ${ }^{\text {a }}(n=84)$} & \multicolumn{3}{|c|}{ PTEN cytoplasmic expression $^{\text {a }}(n=85)$} & \multicolumn{3}{|c|}{ PTEN nuclear expression ${ }^{\mathbf{a}}(n=85)$} \\
\hline & $\begin{array}{c}\text { Low } \\
\text { expression, } \\
S \leq 1\end{array}$ & $\begin{array}{c}\text { High } \\
\text { expression, } \\
S \geq 2\end{array}$ & $P$ value & $\begin{array}{c}\text { Low } \\
\text { expression, } \\
S \leq 1\end{array}$ & $\begin{array}{c}\text { High } \\
\text { expression, } \\
S \geq 2\end{array}$ & $P$ value & $\begin{array}{c}\text { Low } \\
\text { expression, } \\
S \leq 1\end{array}$ & $\begin{array}{c}\text { High } \\
\text { expression, } \\
S \geq 2\end{array}$ & $P$ value \\
\hline $\begin{array}{l}\text { MTC } \\
\text { (all cases) }\end{array}$ & $39(46.4 \%)$ & $45(53.6 \%)$ & - & $50(58.8 \%)$ & $35(41.2 \%)$ & - & $64(75.3 \%)$ & $21(24.7 \%)$ & - \\
\hline$R E T \mathrm{WT}^{\mathrm{b}}$ & $18(46.2 \%)$ & $21(53.8 \%)$ & 1.000 & $24(58.5 \%)$ & $17(41.5 \%)$ & 1.000 & $29(70.7 \%)$ & $12(29.3 \%)$ & 0.452 \\
\hline $\begin{array}{l}R E T \\
\quad \text { mutation }\end{array}$ & $21(46.7 \%)$ & $24(53.3 \%)$ & & $26(59.1 \%)$ & $18(40.9 \%)$ & & $35(79.5 \%)$ & $9(20.5 \%)$ & \\
\hline$R A S W^{\mathrm{b}}$ & $38(52.1 \%)$ & $35(47.9 \%)$ & 0.009 & $47(63.5 \%)$ & $27(36.5 \%)$ & 0.045 & $57(77.0 \%)$ & $17(23.0 \%)$ & 0.453 \\
\hline $\begin{array}{l}\text { RAS } \\
\quad \text { mutation }\end{array}$ & $1(9.1 \%)$ & $10(90.9 \%)$ & & $3(27.3 \%)$ & $8(72.7 \%)$ & & $7(63.6 \%)$ & $4(36.4 \%)$ & \\
\hline
\end{tabular}

$P \leq 0.05$ where considered significant.

${ }^{a} p$-S6 and PTEN intensity expression was stratified in two groups, according to its intensity score - low expression when intensity score was 0 or 1 and high expression for scores of 2 or 3. $\chi^{2}$ tests were performed to analyse the differences in proteins expression in mutated vs wild type (WT) cases for RET and RAS. ${ }^{b} R E T$ WT group includes the cases mutated for RAS. In the same way, RAS WT group includes cases mutated for RET. 
tumors $(P=0.042)$. No significant association was observed between p-S6 expression and age, tumor size and presence of amyloid stroma (Table 2).

\section{PTEN immunohistochemical expression in MTC}

After observing that p-S6 is highly expressed in MTC, we studied PTEN expression by IHC assays in an attempt to clarify whether the expression of this mTOR pathway inhibitor could be associated with the aforementioned findings. We observed total absence of PTEN expression in three cases $(3.5 \%)$. High PTEN cytoplasmic expression $(S \geq 2)$ was present in $41.2 \%(35 / 85)$ of the tumors, whereas $24.7 \%(21 / 85)$ of the MTC disclosed high PTEN nuclear intensity. In $41.2 \%(35 / 85)$ of the cases, no nuclear PTEN expression was detected $(S=0)$ (Tables 2 and 3).

No significant correlation was found between RET mutational status and PTEN expression intensity or localisation. At variance with this, we observed a significantly lower cytoplasmic PTEN expression in WT RAS cases than in cases with $R A S$ mutation $(P=0.045$; Table 3 and Fig.1).

No significant associations were found regarding clinicopathological features and PTEN expression or localisation (Table 2).

\section{Discussion}

The recent description of $H R A S$ and KRAS mutations in sporadic MTC by Moura et al. has shaken the $R E T$ paradigm in MTCs and contributed to relaunch the interest in the molecular study of sporadic MTCs. In this study, we identified the presence of RAS mutations in $12.6 \%$ of the whole series of MTC $(14.3 \%$ of the sporadic cases and $28.6 \%$ of the RET-negative cases), and confirmed that $R A S$ mutations occur in a mutually exclusive pattern with RET mutation. Besides, this, RET mutations were present in $52.9 \%$ of the MTC, thus reinforcing the prominent role of such mutations in the development of hereditary and sporadic MTC. We confirmed the hereditary nature of the five suspected cases and found RET germinative mutations in five apparently sporadic MTCs. These figures are in accordance with previous studies in MTC (11).

We think that the most interesting results of our study concern the mTOR pathway activation in MTC $(13,15,17)$. In a previous study, mTOR pathway expression was found to be frequently detected in MTC, namely in MTC metastatic lesions (13). In our series,
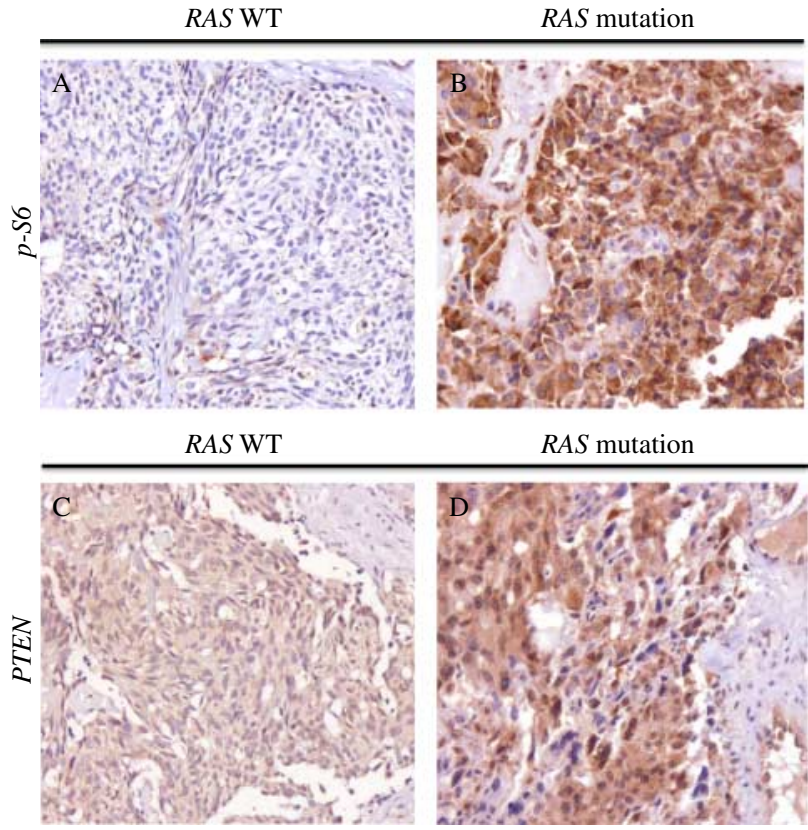

\section{Figure 1}

p-S6 and PTEN expression in tumours with and without RAS mutation. (A) Low $\mathrm{p}$-S6 expression in a WT RAS tumour (intensity score $\leq 1$ ); (B) high $\mathrm{p}$-S6 expression in a tumour with RAS mutation (intensity score $\geq 2$ ); (C) low PTEN expression in a WT RAS tumour (intensity score $\leq 1$ ); and (D) high PTEN expression in a RAS mutated tumour (intensity score $\geq 2$ ). A full colour version of this figure available via http://dx.doi.org/ 10.1530/EJE-14-0389.

we observed mTOR activation in the majority of the tumors, $54.8 \%$ of the cases showing high p-S6 expression. mTOR activation in MTC is not surprising since RET oncogene is known to promote the activation of RAS/MAPKK/extracellular signal-related kinase (RAS/MEK/ ERK) pathway and the phosphoinositide 3-kinase (PI3K)/ AKT/mTOR pathway, controlling cell proliferation, invasion and survival (13).

Like Tamburrino et al. (13) we did not find any association between germinative or sporadic RET mutations and p-S6 expression. In contrast to this, Rapa et al. (15) reported that phospho-mTOR, phospho-AKT, and phospho-p70S6K expression correlated positively with the presence of germline RET mutations. The discrepancies between the results obtained in our and Tamburrino et al. (13) studies and those reported by Rapa et al. (15) may be due to the different elements of mTOR cascade used to evaluate its activity in the three studies. Rapa et al. (15) did not evaluate p-S6 protein as we and 


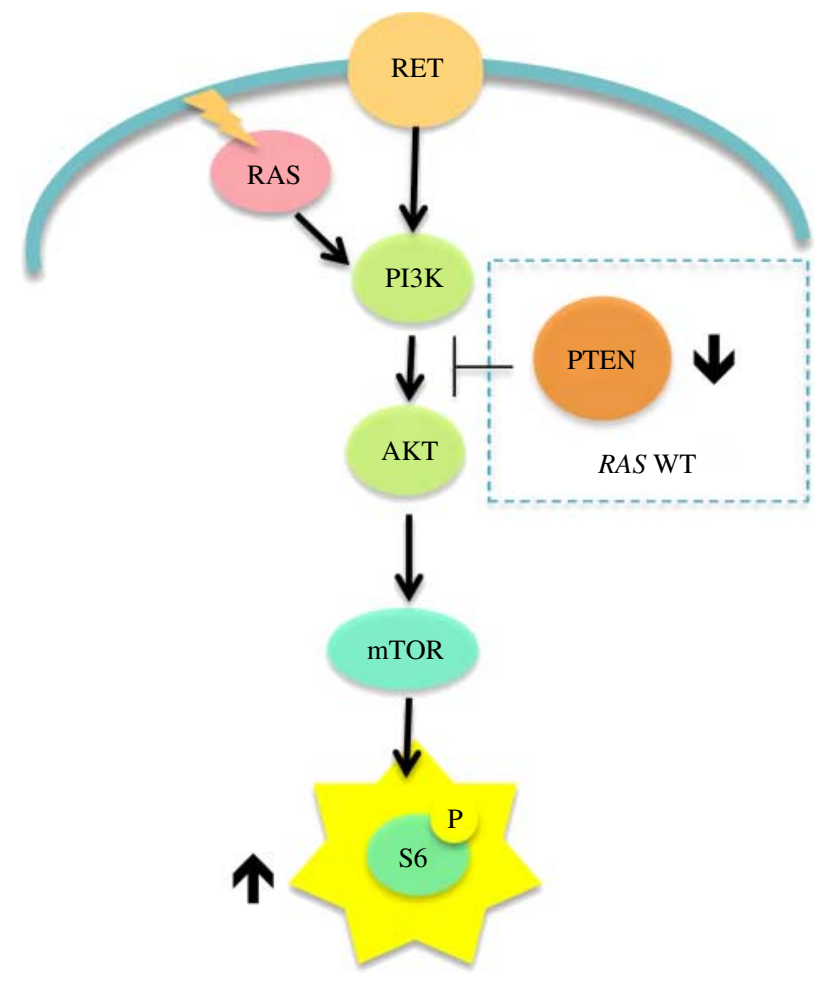

\section{Figure 2}

Schematic representation of mTOR pathway activation in MTC according to RET and RAS mutational status. mTOR activation seems to be a common feature of MTC. High expression levels of p-S6 can result from RAS or RET mutation, as well as from low levels of cytoplasmic PTEN in WT RAS tumours. A full colour version of this figure available via http://dx.doi.org/10.1530/EJE14-0389.

Tamburrino et al. (13) did; in contrast they focused their study on upstream elements of the mTOR pathway (15). Also, the size of the series and the relative composition in terms of sporadic and hereditary RET-mutated cases can underlie those differences. Larger series are needed to fully clarify these aspects. Despite these differences, in all the three studies, the prominent expression of mTOR pathway proteins is a recurrent finding in MTC.

Interestingly, we observed that $R A S$ mutation was significantly associated with higher intensity of p-S6 expression $(P=0.007)$. To our knowledge, this is the first study reporting p-S6 evaluation in RAS-mutated MTCs. $R A S$ itself stimulates numerous intracellular transducers, including PI3K, which is part of mTOR cascade and, as so, could mediate the activation we observed in MTCs with RAS mutation (18, 19; Fig.2).
In our series, p-S6 expression was significantly higher in invasive tumors and in tumors presenting lymph node metastases, a finding that supports the results reported by Tamburrino et al. (13) who described an increased p-S6 expression in metastases from MTC in comparison with autologous matched primary MTCs. Altogether, these results suggest that p-S6 expression can be an indicator of increased metastatic capacity of MTC.

Another interesting result of this study concerns the possible dissociation between RAS mutation and $\mathrm{pS} 6$ expression with regard to the invasive capacity of MTCs. It seems that the overexpression of pS6, regardless of the presence of $R A S$ mutation or its absence, is associated with a significantly higher invasive capacity of MTCs. The study of a larger series with more cases with and without RAS mutation and detailed clinical information is necessary to confirm the aforementioned putative role of p-S6 as a biomarker of MTC aggressiveness.

The contribution of PTEN, an inhibitor of mTOR pathway, has not been addressed before, in MTC, to the best of our knowledge. The analysis of PTEN in our series revealed that PTEN expression was present in almost every tumor (only three cases were negative for this protein). We observed that a significant lower cytoplasmic expression of PTEN was present in WT RAS cases $(P=0.045)$. Taking into consideration that approximately half $(35 / 73-47.9 \%)$ of WT RAS cases showed high p-S6 expression, the aforementioned decrease in PTEN expression may serve as an alternative mechanism for mTOR pathway activation. Indeed, when we consider only the tumors with high p-S6 expression and compare WT RAS- and RAS-mutated tumors, there is a significant association of low PTEN expression with WT RAS cases (62.9\% of WT RAS cases vs $20.0 \%$ in RAS mutated; $P=0.029$ ). This result suggests that low PTEN expression is not a central mechanism of mTOR pathway activation in $R A S$-mutated cases, but it may be crucial to drive mTOR activation in WT RAS tumors.

Loss of PTEN function is not only related to mTOR pathway activation. It has been proposed that PTEN may also play a role inside the nucleus, where it regulates chromosomal integrity, cell cycle progression, p53 acetylation, and DNA-damage response through the interaction with RAD51 as well as in apoptosis induction (20). Putting our results together it seems that the mechanisms of action of PTEN may be twofold: on one hand the decrease in PTEN cytoplasmic expression can lead to a higher mTOR activity, and on the other hand, the high nuclear PTEN expression observed in some of our cases can also influence other cellular processes and putatively contribute to 
tumorigenesis. Further studies are necessary to evaluate whether or not PTEN plays a major role in this setting.

It has been demonstrated that MTC cancer cell lines are sensitive to mTOR inhibitors $(3,4,13,21)$. It was also observed, in melanoma $(22,23)$, that sensitivity to an mTOR inhibitor was correlated with pre-treatment p-S6 levels, but not with other downstream effectors of this pathway (PI3K and p-AKT) $(22,23)$. Similarly, in a phase II study of everolimus in gastric cancer refractory to chemotherapy, higher $\mathrm{p}-\mathrm{S} 6^{\text {Ser240/4 }}$ expression was significantly associated with a higher rate of disease control and achievement of clinical response or stable disease. Therefore, p-S6 expression can be a potential predictive biomarker for everolimus response (24). Further studies are necessary to verify whether p-S6 expression in MTC may represent a predictive sensitivity biomarker to MTOR inhibitors.

Summing up, we report, for the first time, an association between the activation of the mTOR pathway and the presence of RAS mutations in MTCs. Further studies are needed to confirm the usefulness of p-S6 expression as an indicator of invasive capacity of MTC and as a predictive biomarker for the treatment of MTC patients with mTOR inhibitors.

\section{Supplementary data}

This is linked to the online version of the paper at http://dx.doi.org/10.1530/ EJE-14-0389.

\section{Declaration of interest}

The authors declare that there is no conflict of interest that could be perceived as prejudicing the impartiality of the research reported.

\section{Funding}

This study was supported by the Portuguese Foundation for Science and Technology through PhD grant to J Vinagre (Ref: SFRH/BD/81940/2011). Further funding was obtained from the project 'Microenvironment, metabolism and cancer' that was partially supported by ProgramaOperacional Regional do Norte (ON.2 - O Novo Norte) under the Quadro de ReferênciaEstratégicoNacional (QREN) and the Fundo Europeu de Desenvolvimento Regional (FEDER). Ipatimup is an Associate Laboratory of the Portuguese Ministry of Science, Technology, and Higher Education that is partially supported by the FCT.

\section{Author contribution statement}

J Lyra, V Pinto, R Batista, and J Vinagre performed the experimental work (mutational and IHC analysis). M Sobrinho-Simões, C Eloy, and J Lyra performed the histological review of the cases and immunohistochemical evaluation. H Prazeres and $\mathrm{F}$ Rodrigues collect cases and clinical information. P Soares supervised the study, analysed the data, and wrote the first draft together with J Lyra. M Sobrinho-Simões performed a critical review of the MS. All the authors contributed to the writing of the final version.

\section{Acknowledgements}

The authors thank Astrazeneca - Portugal for partial funding of this study through a research project. The funders had no role in the study design, data collection and analysis, interpretation of the results, the preparation of the manuscript or the decision to submit the manuscript for publication.

\section{References}

1 Jarzab B \& Feldt-Rasmussen U. Introduction to European comments on "medullary thyroid cancer: management guidelines of the American Thyroid Association“. Thyroid Research 20136 (Suppl 1) S1. (doi:10.1186/1756-6614-6-S1-S9)

2 Schlumberger M, Carlomagno F, Baudin E, Bidart JM \& Santoro M. New therapeutic approaches to treat medullary thyroid carcinoma. Nature Clinical Practice Endocrinology \& Metabolism 2008 4 22-32. (doi:10.1038/ ncpendmet0717)

3 Grozinsky-Glasberg S, Rubinfeld H, Nordenberg Y, Gorshtein A, Praiss M, Kendler E, Feinmesser R, Grossman AB \& Shimon I. The rapamycin-derivative RAD001 (everolimus) inhibits cell viability and interacts with the Akt-mTOR-p70S6K pathway in human medullary thyroid carcinoma cells. Molecular and Cellular Endocrinology 2010315 87-94. (doi:10.1016/j.mce.2009.09.027)

4 Prazeres H, Torres J, Rodrigues F, Couto JP, Vinagre J, SobrinhoSimoes M \& Soares P. How to treat a signal? Current basis for RET-genotype-oriented choice of kinase inhibitors for the treatment of medullary thyroid cancer. Journal of Thyroid Research 20112011 678357. (doi:10.4061/2011/678357)

5 Couto JP, Almeida A, Daly L, Sobrinho-Simoes M, Bromberg JF \& Soares P. AZD1480 blocks growth and tumourigenesis of RET-activated thyroid cancer cell lines. PLoS ONE 20127 e46869. (doi:10.1371/ journal.pone.0046869)

6 Almeida MQ \& Hoff AO. Recent advances in the molecular pathogenesis and targeted therapies of medullary thyroid carcinoma. Current Opinion in Oncology 201224 229-234. (doi:10.1097/CCO. Ob013e328351c71a)

7 Prazeres HJ, Rodrigues F, Figueiredo P, Naidenov P, Soares P, Bugalho MJ, Lacerda M, Campos B \& Martins TC. Occurrence of the Cys611Tyr mutation and a novel Arg886Trp substitution in the RET proto-oncogene in multiple endocrine neoplasia type 2 families and sporadic medullary thyroid carcinoma cases originating from the central region of Portugal. Clinical Endocrinology 200664 659-666. (doi:10.1111/j.1365-2265.2006.02524.x)

8 Taccaliti A, Silvetti F, Palmonella G \& Boscaro M. Genetic alterations in medullary thyroid cancer: diagnostic and prognostic markers. Current Genomics 201312 1-8. (doi:10.2174/138920211798120835)

9 Elisei R, Cosci B, Romei C, Bottici V, Renzini G, Molinaro E, Agate L, Vivaldi A, Faviana P, Basolo F et al. Prognostic significance of somatic RET oncogene mutations in sporadic medullary thyroid cancer: a 10-year follow-up study. Journal of Clinical Endocrinology \& Metabolism 200893 682-687. (doi:10.1210/jc.2007-1714)

10 Boichard A, Croux L, Al Ghuzlan A, Broutin S, Dupuy C, Leboulleux S, Schlumberger M, Bidart JM \& Lacroix L. Somatic RAS mutations occur in a large proportion of sporadic RET-negative medullary thyroid carcinomas and extend to a previously unidentified exon. Journal of Clinical Endocrinology \& Metabolism 201297 E2031-E2035. (doi:10.1210/jc.2012-2092)

11 Ciampi R, Mian C, Fugazzola L, Cosci B, Romei C, Barollo S, Cirello V, Bottici V, Marconcini G, Rosa PM et al. Evidence of a low prevalence of RAS mutations in a large medullary thyroid cancer series. Thyroid 2013 23 1-26. (doi:10.1089/thy.2012.0207)

12 Moura MM, Cavaco BM, Pinto AE \& Leite V. High prevalence of RAS mutations in RET-negative sporadic medullary thyroid carcinomas. 
Journal of Clinical Endocrinology \& Metabolism 201196 E863-E868. (doi:10.1210/jc.2010-1921)

13 Tamburrino A, Molinolo AA, Salerno P, Chernock RD, Raffeld M, Xi L, Gutkind JS, Moley JF, Wells SA Jr \& Santoro M. Activation of the mTOR pathway in primary medullary thyroid carcinoma and lymph node metastases. Clinical Cancer Research 201218 3532-3540. (doi:10.1158/ 1078-0432.CCR-11-2700)

14 Agrawal N, Jiao YC, Sausen M, Leary R, Bettegowda C, Roberts NJ, Bhan S, Ho AS, Khan Z, Bishop J et al. Exomic sequencing of medullary thyroid cancer reveals dominant and mutually exclusive oncogenic mutations in RET and RAS. Journal of Clinical Endocrinology and Metabolism 201398 E364-E369. (doi:10.1210/jc.2012-2703)

15 Rapa I, Saggiorato E, Giachino D, Palestini N, Orlandi F, Papotti M \& Volante M. Mammalian target of rapamycin pathway activation is associated to RET mutation status in medullary thyroid carcinoma. Journal of Clinical Endocrinology \& Metabolism 201196 2146-2153. (doi:10.1210/jc.2010-2655)

16 Pitt SC \& Chen H. The phosphatidylinositol 3-kinase/akt signaling pathway in medullary thyroid cancer. Surgery 2008144 721-724. (doi:10.1016/j.surg.2008.06.028)

17 Kouvaraki MA, Liakou C, Paraschi A, Dimas K, Patsouris E, Tseleni-Balafouta S, Rassidakis GZ \& Moraitis D. Activation of mTOR signaling in medullary and aggressive papillary thyroid carcinomas. Surgery 2011150 1258-1265. (doi:10.1016/j.surg.2011.09.022)
18 Cerrato A, De Falco V \& Santoro M. Molecular genetics of medullary thyroid carcinoma: the quest for novel therapeutic targets. Journal of Molecular Endocrinology 200943 143-155. (doi:10.1677/JME-09-0024)

19 Hay N \& Sonenberg N. Upstream and downstream of mTOR. Genes and Development 200418 1926-1945. (doi:10.1101/gad.1212704)

20 Salmena L, Carracedo A \& Pandolfi PP. Tenets of PTEN tumour suppression. Cell 2008133 403-414. (doi:10.1016/j.cell.2008.04.013)

21 Faggiano A, Ramundo V, Dicitore A, Castiglioni S, Borghi MO, Severino R, Ferolla P, Crino L, Abbruzzese A, Sperlongano P et al. Everolimus is an active agent in medullary thyroid cancer: a clinical and in vitro study. Journal of Cellular and Molecular Medicine 201216 1563-1572. (doi:10.1111/j.1582-4934.2011.01438.x)

22 Aziz SA, Davies M, Pick E, Zito C, Jilaveanu L, Camp RL, Rimm DL, Kluger Y \& Kluger HM. Phosphatidylinositol-3-kinase as a therapeutic target in melanoma. Clinical Cancer Research 200915 3029-3036. (doi:10.1158/1078-0432.CCR-08-2768)

23 Populo H, Soares P, Rocha AS, Silva P \& Lopes JM. Evaluation of the mTOR pathway in ocular (uvea and conjunctiva) melanoma. Melanoma Research 201020 107-117. (doi:10.1097/CMR.0b013e32832ccd09)

24 Yoon DH, Ryu MH, Park YS, Lee HJ, Lee C, Ryoo BY, Lee JL, Chang HM, Kim TW \& Kang YK. Phase II study of everolimus with biomarker exploration in patients with advanced gastric cancer refractory to chemotherapy including fluoropyrimidine and platinum. British Journal of Cancer 2012106 1039-1044. (doi:10.1038/bjc.2012.47)

Received 14 May 2014

Revised version received 24 July 2014

Accepted 26 August 2014 